\title{
Research on Intellectual Property Risk in the Process of Knowledge
}

\section{Sharing in Cooperative Organizations}

\author{
Sun Yi
}

China Jiliang University

\begin{abstract}
Keywords: knowledge sharing, intellectual property risk
\end{abstract}
\begin{abstract}
In recent years, the form of cooperative organizations formed between the enterprises has become the realistic choice of seeking the sustained competitiveness for many enterprises. Knowledge sharing and information exchange are the key factors of cooperative organizations' success on the premise of knowledge sharing. The original intention of cooperative organizations formed between the enterprises is to increase the flexibility of competition and reduce the market risk, but in the cooperation the enterprises need to face the intellectual property risk. Knowledge is the enterprises' resources with the most strategic importance at present. Therefore, we should analyze and study the main influence factors of intellectual property risk for influencing the cooperative organizations, evaluate the enterprise risk management level and strengthen the management and protection of enterprises' intellectual property. This undoubtedly has important practical significance.
\end{abstract}

\section{Introduction}

Once the intellectual property risk created, the loss caused by it is huge. Therefore, in the process of cooperative innovation, we must pay attention to the intellectual property risk in the knowledge sharing and carry on the effective management. Scientifically analyzing and evaluating the risk factors and the risk intensity of influencing the intellectual property is the necessary condition. The intellectual property risk assessment refers to that we can obtain the occurrence probability of the intellectual property risk events caused by the single risk factor and the size of negative effect degree by discerning the possible risk factors of infringing upon intellectual property in the process of enterprises' knowledge sharing and according to the identified risk factors and combined with the quantitative and qualitative analysis method. We can also analyze the overall risk occurrence level status and the level of occurrence risk of the intellectual property in knowledge sharing further. Finally, based on the analysis of the risk assessment and combined with the related actual situation, we can work out the prevention measures of intellectual property risk in the risk knowledge sharing to realize the management of intellectual property risk in the knowledge sharing. In this paper, our research will provide the basis for reducing the loss of cooperative organizations' interests and realizing a reliable and effective intellectual property risk management.

\section{The Overview of Intellectual Property Risk Factor Analysis}

Having a detailed and accurate analysis on the risk factors of intellectual property in the knowledge sharing is the first step of having the risk assessment successfully and it is also the foundation of later various stages. If we can't accurately identify where the risks are, and explicit the possible relationship between the risks, we can't analyze and predict the crisis for the loss of intellectual property in the cooperative organizations. Of course, we also have no way to formulate 
the countermeasures to control risk. Therefore, in this stage, the risk analysis personnels not only need to widely collect data, have access to information and take advice, and based on this have the detailed and thorough analysis on various related factors of the risk main body and its environment to determine the risk factors, but also need to make many obtained risk factors be classified to clear the hierarchical structure and the logical relation model between risk factors. In this way, it can provide the important basis for the process of forming the risk assessment index system model and realizing the quantitative risk assessment.

The analysis of risk factors is conducted under the condition of unclear, incomplete and inaccurate information, and it is a process of clearing the relationship between the factors. In this case, because it lacks the necessary conditions of reasoning and it is limited by the information and the method, it's difficult to avoid having the speculation ingredients in the process of risk analysis, and the results of the analysis also do not necessarily be completely consistent with the objective fact. However, these methods also reflect the risk state of the risk main body from a certain angle, a certain range, and a certain extent. In the process of concrete analysis, we need to conduct a large number of collection and sorting on the relevant data in the field. These data not only include the theoretical literature data and the technical data involved in this field and related with this field, but also may include the related real case data, and the questionnaires obtained by the expert survey and others. By the analysis of these data, we summarize and induce the risk factors involved in risk investigation. However, whether the risk factors obtained by the expert questionnaire analysis or the literature material and the case analysis summary, we only can conclude and summarize the risk factors involved in the related problems, and we can't make whether there are the category relationship and the hierarchical structure between these risk factors be presented very clearly. It is not quite good for the risk quantitative analysis further. For this reason, we need to make use of the related theories or methods to solve this problem.

\section{The Intellectual Property Risk Prevention in the Knowledge Sharing of Cooperative Organizations}

The Outside Environment Prevention System of Cooperative Organizations. Seen from the big environment, China's intellectual property protection environment is relatively weak. In 2003, the Political and Economic Risk Consultancy in Hong Kong released the intellectual property risk index of main countries and regions in Asia, and the risk index comprehensively reflected a country's intellectual property law protection system. The regulations and policies of intellectual property, the intellectual property management level of government, enterprises and scientific research departments, the national consciousness of intellectual property, the efficiency of relevant government functional organization and others were one of the important comprehensive factors indexes which could measure a country's intellectual property protection level. The index was calculated as ten at the highest level. The closer to zero the level was, it meant that the lower the risk was, and the better the ranking became. However, the closer to zero the index level was, it stood for that the greater the risk was. While China was ranked second in 12 Asian countries and it was as high as 9.34. Thus, the country should urgently need to accelerate the improvements in the modern intellectual property system of "clear ownership, well defined power and responsibility, strict protection and free transferring”. In the process of formulating economic structure, industrial structure adjustment and scientific and ethnological development programme, we should strengthen the study and use the consciousness of intellectual property strategy. For not enough perfect legal system in our country, the internationalized cross-regional cooperation brings the difference of legal system and jurisdiction right, and the difference of cultural background, and the new problems and challenges brought by informatization and networking. Thus, these things can improve the level of intellectual property protection in China on the whole and provide the intellectual property external 
big environment with relatively small risk for the cooperative organization in the knowledge sharing.

The Intellectual Property Risk Prevention and Control System in Advance in Cooperative Organization.

(1) We should select partners and cooperation project, and examine their cooperation intentions, abilities, and reputations: In practice, the enterprises need to have the selection criteria and selection method of cooperative enterprises with the scientific formulation and evaluate the candidate enterprises' cooperative willingness, and completely eradicate the willingness of individual managers to stand for the enterprises' overall willingness, and focus on tracking and preparedness for the enterprises with the bigger risk. We should fully investigate and assess the key resources, the core competences, the advantages and specialties and the value-added role provided by the cooperative partners so as to prevent the harm caused by the information asymmetry. We should fully check on the candidate partners' economic strength and credit record.

(2)We shoulddesign relatively complete cooperation agreement: In the organization, each enterprise needs to appoint the input resources and the relationship of rights and obligations on this basis. The input intellectual property and cooperation will make all the caused intellectual properties be assessed to clear the rights and realize the balance of rights and obligations. We should strengthen and evade the terms of agreement of moral risk and raise the cost of deception to prevent the mutual deception behavior. We should adopt the dynamic contract. We need to clear the mutual contribution of the two parties for the cooperation, and the sharing mechanism of character and innovation in the process of cooperation in the contract. We should clear the processing and compensation mode of having the improper access to knowledge and technology disputes and clear the office and production area which can be arrived in the process of mutual interview, visit and others.

(3) We should do the enterprises' knowledge partition and the level classification protection: We need to sign the confidentiality agreement of core knowledge between partners and strictly abide by it. Concrete content: The two parties should sign the definite protection clause to protect a technology which is only limited within the scope of cooperation innovation and is not allowed to spread to a third party to use. We sign the restrictive clause to make it be used to protect the partners to have the possibility to obtain these technologies by participating in trials, contacting the enterprises' internal documents, talking to employees, and participating in all kinds of report meetings and other ways. For the technologies which the enterprises provide to the cooperative innovation but the cooperating parties are limited to use, the primary means of protection is to only offer those technologies which have applied for and obtained the patent.

The Intellectual Property Risk Prevention System of Cooperative Organizations in the Process of Cooperation.

(1) We should improve the staff technical protection consciousness: We should teach the staffs to know the serious harmfulness of technology loss and tell the staffs to need to pay attention to what technology protection projects in the process of cooperation. The enterprises need to make sure who the possible operation users of developing and applying the core knowledge and technology are in their own enterprises and cooperative organizations. In enterprise staffs' interpersonal communication, the contact persons need to avoid providing the improper technical documentation and talking about the protected technical topics in transactional communication activities. The staffs involved in the research and development need to not disclose the technologies which have nothing to do with the cooperation project or keep outside the shared scope o under the contract. The managerial personnels need to pay attention to the situation that they should not leak the enterprises' technological secrets in the process of negotiating with the other party.

(2) We should conduct the core staffs' flow limit system: In order to prevent the occurrence of brain drain, technologies and other losses caused by this in the process of cooperation, the various cooperative parties should limit the flow of the staffs between the partners in certain period under the terms of a contract. For example, in the process of appointed cooperation, any party shall not hire the staffs from the other party. Without the consent of the other party, any party shall not hire 
the staffs who have been resigned from the cooperating parties in recent several years.

(3) We should build the real-time audit system of intellectual property: In the process of cooperation, the enterprises need to actively check whether the enterprises' technical management system is perfect or not, and whether effectively abide by it or not. We adopt the regular or irregular inspection way to check on the two parties' execution effect of cooperative contract. We should strengthen the communication with the other party, and timely find the knowledges which is possible to lose to the other party, and make the necessary negotiations, and reduce the loss.

(4) We should build the multi-angle credit guarantee incentive mechanism in the internal of cooperation: All parties of cooperation need to design jointly a set of perfect dynamic cooperation internal trust evaluation system to conduct the comprehensive evaluation for each member's past, present and future so as to build the reliable mutual trust mechanism. We should enhance and evade the terms of an agreement to raise the cost of cheating and prevent the behavior of cheating each other. We should give the positive motivation of increasing the income of the cooperation to the behaviors which have the positive cooperation and are beneficial to the trust relationship and the contribution so as to build the enterprise senior management personnels' good interpersonal relationship.

\section{Conclusion}

The research on the intellectual property risk between the cooperative organizations staffs in the process of knowledge sharing is valuable. The problems of intellectual property risk not only exist in the cooperative organizations, and its probability of occurrence is also relatively high. What's more, once its risk occurs, the consequences level is slightly high. Even it will bring the huge losses for the single enterprise and even the whole cooperative organization's knowledge assets and economic benefits. For this reason, we put forward the cooperative organization's external environment protection, organize and build the risk prevention system in advance and the real-time protection system in the process of organization operation. We can establish the risk prevention measurements of intellectual property from the angle of before, during the events for two combinations of the internal and external environment. These contents about the establishment of the intellectual property risk assessment theory model in knowledge sharing, the empirical research and the formulation of countermeasures provide new conception and mode for the research on the knowledge economy era of taking the knowledge as the core resource, the intellectual property, especially the intellectual property risk problems which exist in the knowledge cooperative organization.

\section{References}

[1]Lin Shimin, Tian Fengzhan, and Lu Yulv. Bayesian Network Construction and Its Application in Data Mining.Journal of Tsinghua University (Science and Technology Edition), 2001, 41(l):49- 52.

[2]Cao Dongming, Zhang Boming, and Deng Youman. Research on A Kind of New-type Fault Location Method. Automatic Control on Electrical Power System,1999,23(7):12- 14.

[3]Li Qingdian, etc. Dam Safety Risk Analysis based on Bayesian Network. International Symposium on Water and Electricity in 2006.

[4]Fu Yu, etc. Information Security Risk Assessment Method based on Bayesian Network[J].Journal of Wuhan University (Science Edition) , 2006,52(5). 